\title{
Turbulent power spectrum in warm and cold neutral medium using the Galactic $\mathrm{H}$ I $21 \mathrm{~cm}$ emission
}

\author{
Samir Choudhuri ${ }^{1 \star}$ and Nirupam Roy ${ }^{2}$ \\ 1 National Centre For Radio Astrophysics, Post Bag 3, Ganeshkhind, Pune 411 007, India \\ 2 Department of Physics, Indian Institute of Science, Bangalore 560012, India
}

\begin{abstract}
Small-scale fluctuations of different tracers of the interstellar the medium can be used to study the nature of turbulence in astrophysical scales. Of these, the "continuum" emission traces the fluctuations integrated along the line of sight whereas, the spectral line tracers give the information along different velocity channels as well. Recently, Miville-Deschênes et al. (2016) have measured the intensity fluctuation power spectrum of the continuum dust emission, and found a power law behaviour with a power law index of $-2.9 \pm 0.1$ for a region of our Galaxy. Here, we study the same region using high-velocity resolution 21-cm emission from the diffuse neutral medium, and estimate the power spectrum at different spectral channels. The measured $21-\mathrm{cm}$ power spectrum also follows a power law, however, we see a significant variation in the power law index with velocity. The value of the power-law index estimated from the integrated map for different components are quite different which is indicative of the different nature of turbulence depending on temperature, density and ionization fraction. We also measure the power spectra after smoothing the $21 \mathrm{~cm}$ emission to velocity resolution ranging from 1.03 to $13.39 \mathrm{~km} \mathrm{~s}^{-1}$, but the power spectrum remains unchanged within the error bar. This indicates that the observed fluctuations are dominantly due to density fluctuations, and we can only constrain the power-law index of velocity structure function of $0.0 \pm 1.1$ which is consistent with the predicted Kolmogorov turbulence $(\gamma=2 / 3)$ and also with a shock-dominated medium $(\gamma=1.0)$.
\end{abstract}

Key words: ISM: atoms - ISM: general - ISM: structure - radio lines: ISM - physical data and process: turbulence

\section{INTRODUCTION}

Study of the Galactic neutral hydrogen (H I) $21 \mathrm{~cm}$ emission can reveal the kinematic and morphological properties of the interstellar medium (ISM) of our Galaxy. Several allsky surveys were performed at different angular scales to probe the distribution of $\mathrm{H}$ I within the Galaxy. For example, the Leiden/Argentine/Bonn (LAB) Survey (Kalberla et al. 2005) is one of the most sensitive Milky Way H I surveys which has extensive coverage both spatially and kinematically. Recently, H I4 $\pi$ survey (HI4PI Collaboration et al. 2016) supersedes the LAB survey with a higher angular resolution. Neutral atomic ISM has structures over a wide range of scales. Early observations indicated the presence of cold H I in the form of "clouds" of size few parsec (Field et al. 1969, Field 1973). Further observations with high resolution and sensitivity show the structure of even smaller scales of the order of sub-pc (Crovisier et al. 1985 Kalberla et al. 1985). These structures are believed to be generated

\footnotetext{
^ Email:samir@ncra.tifr.res.in
}

by the compressible turbulence in the ISM. Dieter et al. (1976) have reported variation in the optical depth of interstellar $\mathrm{H}$ I on $70 \mathrm{AU}$ scale in front of a quasar 3C 147 using Very Long Baseline Interferometry (VLBI) observations. Similarly, there are multi-epoch pulsar observations which reveal the opacity variations on several AU scales (Deshpande et al. 1992 Frail et al. 1994, Davis et al. 1996. Johnston et al. 2003, Stanimirović et al. 2010).

Statistical measurements of the power spectrum, $P(\mathrm{k})$ is a powerful technique to probe and quantify the small-scale structures in the ISM. Observations of H I $21 \mathrm{~cm}$ emission have shown power law power spectra on pc scales (Crovisier \& Dickey 1983, Green 1993, Dickey et al. 2001). Roy et al. (2010) and Deshpande et al. (2000) have measured power spectra to quantify the fluctuations even at smaller scales ( AU scale) for different parts of the Galaxy. The structure function of the opacity fluctuations have also been used to study the tiny scale fluctuations. For example, Roy et al. (2012) and Dutta et al. (2014) have used H I absorption to measure the structure-function of the opacity fluctuations down to a few tens of $\mathrm{AU}$ scale, and showed it to have a 
power law scaling. Even if the details are not completely understood, analytical and numerical works (Deshpande 2000 Nagashima et al. 2006, Vázquez-Semadeni et al. 2006. Hennebelle \& Audit 2007) suggest turbulence as the key mechanism for generating these small-scale structures.

From an observational point of view, the $21 \mathrm{~cm}$ emission power spectrum could be modified due to the turbulent velocity fluctuations in the field. Lazarian \& Pogosyan (2000) showed that velocity fluctuations make the estimated power spectra shallower, provided that the velocity width of the individual spectral channel is much smaller than the turbulent dispersion of the $\mathrm{H}$ I gas. To test this, Deshpande et al. (2000) estimated the H I power spectrum towards the directions of Cas $\mathrm{A}$ and Cygnus $\mathrm{A}$ with and without velocity averaging, but they did not find any change in the slope of the measured power spectrum. It indicates that the contribution of the velocity fluctuations is less significant in the measured power spectrum for those directions. Later, Roy et al. (2010) have also measured the opacity power spectrum in the direction of Cas A with better velocity resolution, and they also have not found any change in the power law index due to velocity averaging.

In this paper, we study the $\mathrm{H}$ I $21 \mathrm{~cm}$ power spectrum in the direction of Galactic coordinate $(l, b)=\left(198^{\circ}, 32^{\circ}\right)$. For this direction, Miville-Deschênes et al. (2016) have used the Planck radiance map at $857 \mathrm{GHz}$, the MegaCam g band map and the WISE $12 \mu \mathrm{m}$ emission map to estimate the dust continuum emission power spectrum. Combining these three observations, they measured the spatial power spectrum over a large range of scales ranging from $0.01 \mathrm{pc}$ to $50 \mathrm{pc}$. They found a single power law with index $-2.9 \pm 0.1$, consistent with the density field power spectrum of turbulent neutral ISM. They have given some estimate of the scale, less than $0.01 \mathrm{pc}$, at which the turbulent energy dissipates. Here, we use the spectral information of $\mathrm{H}$ I $21 \mathrm{~cm}$ emission for power spectrum estimation for narrow velocity channels. Our aims here are: (a) to measure the variation of turbulence power spectra in different velocity channels, and (b) to quantify the fluctuations of the velocity structures, if present, by estimating power spectrum for different velocity resolution. We use the high-velocity resolution LAB survey (Kalberla et al. 2005) data for this purpose.

A brief outline of the paper follows. Data analysis is presented in Section 2. In Section 3, we present the results of the power spectrum measurement. Finally, we summarize and conclude in Section 4.

\section{DATA ANALYSIS}

LAB survey presents all-sky $21 \mathrm{~cm}$ emission spectra by combining the Leiden/Dwingeloo Survey (Hartmann \& Burton 1997) and the Instituto Argentino de Radioastronoma Survey (Arnal et al. 2000 Bajaja et al. 2005) data. The angular resolution of this survey is around half a degree. The velocity range covers $-450 \mathrm{~km} \mathrm{~s}^{-1}$ to $400 \mathrm{~km} \mathrm{~s}^{-1}$ with a channel separation $1.03 \mathrm{~km} \mathrm{~s}^{-1}$. The rms brightness temperature of the survey is $0.07-0.09 \mathrm{~K}$.

We use the NRAO Astronomical Image Processing System (AIPS) package for further analysis of the LAB survey data. We select a region centred at Galactic coordinate $\left(198^{\circ}, 32^{\circ}\right)$ from the LAB survey for our purpose. Figure

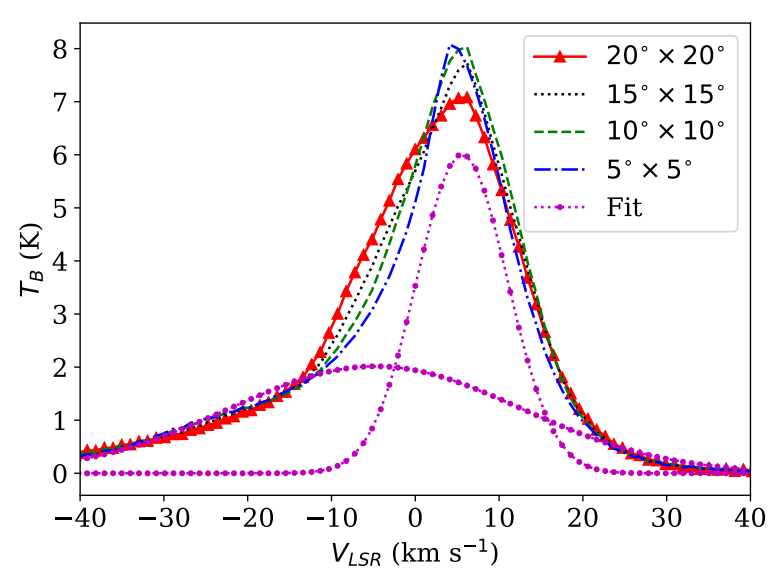

Figure 1. The H I $21 \mathrm{~cm}$ spectra averaged over different spatial dimensions centred on Galactic coordinate $\left(198^{\circ}, 32^{\circ}\right)$. The magenta circles show the two components Gaussian fits to the spatially averaged spectra from the central $5^{\circ} \times 5^{\circ}$ region.

1 shows the spatially average spectra over a velocity range of $-40 \mathrm{~km} \mathrm{~s}^{-1}$ to $+40 \mathrm{~km} \mathrm{~s}^{-1}$. The red solid line with triangles shows the spectra averaged over angular dimensions $20^{\circ} \times 20^{\circ}$. The peak value of this spectra is around $8 \mathrm{~K}$ at a velocity $+4 \mathrm{~km} \mathrm{~s}^{-1}$. The black dotted, green dashed, and blue dash-dot lines show different spectra averaged over an image of dimensions $15^{\circ} \times 15^{\circ}, 10^{\circ} \times 10^{\circ}$ and $5^{\circ} \times 5^{\circ}$ respectively. Please note that the spectra are not symmetric along the positive and negative velocity ranges with respect to the peak. There is some wide emission component in the negative velocity region whereas the spectra fall relatively more sharply in positive velocity range. The best twocomponent Gaussian fit (magenta circles) of the averaged spectra from the central $5^{\circ} \times 5^{\circ}$ region indicates the presence of two distinct phases: (a) the cold phase (Cold Neutral Medium; CNM) with smaller width $\left(\sigma_{v}=5.4 \mathrm{~km} \mathrm{~s}^{-1}\right)$, and (b) the warm phase (Warm Neutral Medium; WNM) with significantly larger width $\left(\sigma_{v}=17.3 \mathrm{~km} \mathrm{~s}^{-1}\right)$. In Figure 1 we see that the negative velocity range is dominated by the WNM whereas the positive velocity range has more contribution from the CNM. We divide the velocity range -30 to $+20 \mathrm{~km} \mathrm{~s}^{-1}$ with four different regions. The velocity range from -30 to $-15 \mathrm{~km} \mathrm{~s}^{-1}$ can be interpreted as an intermediate-velocity gas component (IVC) as shown in Figure 4 of Miville-Deschênes et al. (2016). The velocity range from -14 to $-5 \mathrm{~km} \mathrm{~s}^{-1}$ is mainly dominated by the WNM of the Galaxy disk. The CNM is dominated in the velocity range from -4 to $+15 \mathrm{~km} \mathrm{~s}^{-1}$. The velocity range $\geq+16 \mathrm{~km} \mathrm{~s}^{-1}$ is again dominated by the WNM, but the relative amplitude is smaller here as compared to the WNM in the negative velocity range. In the next section, we use the $20^{\circ} \times 20^{\circ}$ image with velocity ranges from -30 to $+20 \mathrm{~km} \mathrm{~s}^{-1}$ to study the statistical properties of these components in terms of the power spectrum. We use this velocity span because the power spectrum of brightness temperature fluctuations becomes gradually more noise dominated.

We also measure the power spectrum after averaging the velocity channels of different width. For this purpose, we smooth the cube with a Boxcar function along the frequency by changing the width of the function. We use " $n$ "-pixel 
Turbulence in warm and cold neutral medium

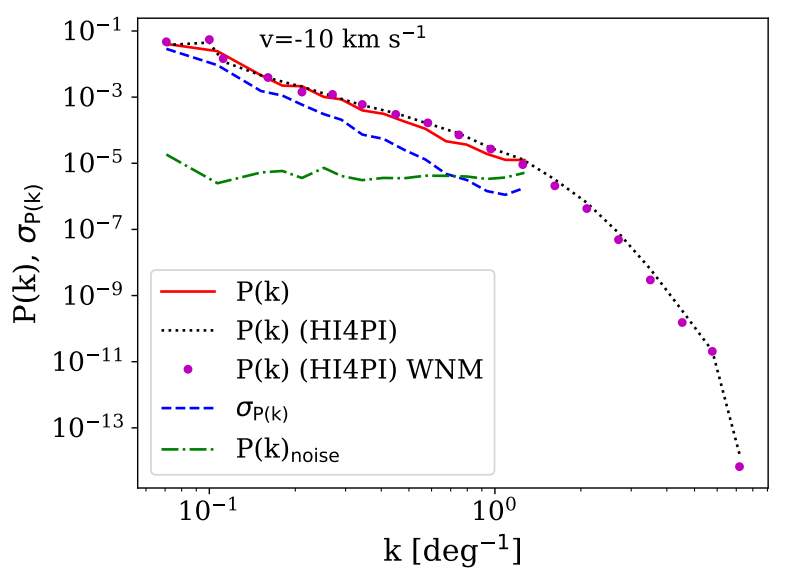

Figure 2. The estimated power spectrum, $P(\mathrm{k})$, for $v_{L S R}=$ $-10 \mathrm{~km} \mathrm{~s}^{-1}$ which is mainly dominated by WNM (red solid line). For comparison, we also show the estimated $P(\mathrm{k})$ for a line free channel which is mainly dominated by the system noise (green dash-dot line). Note that the noise power spectrum is much lower than the $21 \mathrm{~cm}$ signal power spectrum. The measured $P(\mathrm{k})$ for $v_{L S R}=-10 \mathrm{~km} \mathrm{~s}^{-1}$ using $\mathrm{H} \mathrm{I} 4 \pi$ Survey is also shown by black dotted line. The blue dash line shows the expected cosmic variance for this channel assuming the signal to be Gaussian random in nature. The magenta points show the velocity integrated power spectrum in the WNM dominated region $\left(-14<v_{L S R}<-5 \mathrm{~km} \mathrm{~s}^{-1}\right)$ for $\mathrm{H} \mathrm{I} 4 \pi$ data which is remain same as for the single channel power specturm.

$(\mathrm{n}=3,5,7, \ldots)$ boxcar smoothing function centred on each frequncy channel to make a lower resolution cube. We first use AIPS task TRANS and then XSMTH for smoothing. The channel separation of LAB data is $1.03 \mathrm{~km} \mathrm{~s}^{-1}$, whereas the resolution of the LAB survey is $1.25 \mathrm{~km} \mathrm{~s}^{-1}$ (Kalberla et al. 2005. Table 1). The channel resolution for this velocity smoothing will be slightly more than " $n$ " times channel separation due to this correlation. While considering the variation of power spectra after smoothing, we consider channels separated by " $n$ " pixels to consider only independent measurements. We use velocity averaged cubes for the power spectrum measurement in Section 3

\section{POWER SPECTRUM ANALYSIS}

We use the Fastest Fourier Transform in the West (FFTW; Frigo et al. 2005) to convert the image in the Fourier domain and then to estimate the power spectrum at different Fourier modes. To avoid ringing due to sharp cut-off at the edge, we multiply the image with a cosine function. Also, we average azimuthally to increase the signal to noise (SNR) in the final power spectrum estimation. Kalberla \& Kerp 2016 Kalberla et al.2017 have studied the anisotropy in the power spectrum for HI gas distribution. But, we have not seen any significant anisotropies in the measured power spectrum for this field. So, we have considered only the azimuthally averaged power spectrum in the rest of the paper. As mentioned earlier, we use an image of dimensions $20^{\circ} \times 20^{\circ}$ with angular resolution $0.5^{\circ}$ for this purpose. We divide the whole k-range $\left(0.07 \leq \mathrm{k} \leq 1.2 \mathrm{deg}^{-1}\right)$ into 20 equally spaced logarithmic bins to increase the SNR in the estimated $P(\mathrm{k})$.
Assuming that the physical distance of the $\mathrm{H}$ I clouds is 200 pc (Miville-Deschênes et al. 2016), here we probe the fluctuations of length scale ranging from $3 \mathrm{pc}$ to $50 \mathrm{pc}$.

In Figure 2 we show the measured $P(\mathrm{k})$ as a function of $\mathrm{k}$ (in $\mathrm{deg}^{-1}$ ) at LSR velocity $v_{L S R}=-10 \mathrm{~km} \mathrm{~s}^{-1}$ which is mainly dominated by the WNM (red solid line). The green dashed-dot line shows the measured $P(\mathrm{k})$ due to the system noise for a line free channel. We see that the noise power spectrum is roughly constant at different Fourier modes and the magnitude is much lower than the line channel power spectrum. Assuming the signal to be Gaussian in nature, we estimate the expected cosmic variance in the power spectrum measurement which is shown by the blue dashed line. Although the assumption may not be true for Galactic H I signal, we have used this approximation for error estimation in the rest of the paper because we don't otherwise have any analytical formula for estimating the error from a single realization of the signal. In Figure 2, we also show the estimated $P(\mathrm{k})$ at $v_{L S R}=-10 \mathrm{~km} \mathrm{~s}^{-1}$ using $\mathrm{H} I 4 \pi$ survey (black dotted line) for a comparison. As the spatial resolution is higher for $\mathrm{H}$ I $4 \pi$ Survey, we can probe larger $\mathrm{k}$ range $\sim 6$ times more than the LAB survey. For the overlapping range, the two spectra are consistent with each other. However, the amplitude of the power spectrum for $\mathrm{H} \mathrm{I} 4 \pi$ falls significantly at $\mathrm{k} \geq 1.5 \mathrm{deg}^{-1}$. As shown in Figure 2 of Miville-Deschênes et al. (2016), this deviation at smaller angular scales is due to the beam shape of the H I4 $\pi$ survey data. However, the measured power spectrum behaves as a power law for the whole range probed by the LAB survey data. It is due to fact that the pixel size is roughly similar to the beam size for the LAB survey. For the purpose of the present analysis, we restrict ourselves only to LAB survey data. The magenta points in this figure show the velocity integrated power spectrum in the WNM dominated region $\left(-14<v_{L S R}<-5 \mathrm{~km} \mathrm{~s}^{-1}\right)$ for $\mathrm{H} \mathrm{I} 4 \pi$ data. For this case, first, we integrate the image over the mentioned velocity range and then calculate the power spectrum. We see that the integrated power spectrum remains the same as for the single channel power spectrum at $v_{L S R}=-10 \mathrm{~km} \mathrm{~s}^{-1}$ (discussed later). In terms of the spectral resolution, LAB survey provides higher resolution data than $\mathrm{H}$ I $4 \pi$ survey. As the spectral resolution is more important for achieving the main goals of this work, we have used only LAB survey data in the rest of the analysis.

Figure 3 shows the measured $P(\mathrm{k})$ as a function of $\mathrm{k}$ for different velocity channels. In this figure, we show only three representative velocity channels $-22.67,+9.28$ and $+18.54 \mathrm{~km} \mathrm{~s}^{-1}$ which are dominated by IVC, CNM and WNM respectively. In Figure 2 we show another WNM dominated velocity channel from negative velocity range $\left(-10 \mathrm{~km} \mathrm{~s}^{-1}\right)$. The blue dashed lines in each panel show the estimated $P(\mathrm{k})$ as a function of wavenumber $\mathrm{k}$. We fit a power law model $P^{M}(\mathrm{k})=A \mathrm{k}^{\beta}$ to the measured $P(\mathrm{k})$ and the red solid lines show the best-fit power law for each velocity channel. The $k$ range we used for fitting is 0.3 to $0.9 \mathrm{deg}^{-1}$ which are shown by two vertical lines in this figure. The values of the power law index $(\beta)$ are also shown in each panel. We see the slope of the CNM dominated region (middle panel) is steeper as compared to other regions.

We now study the variation of $\beta$ as a function of $v_{L S R}$ (Figure 4). The blue points show the estimated $\beta$ with $1 \sigma$ error bar as a function of $v_{L S R}$. The values of $\beta$ vary be- 

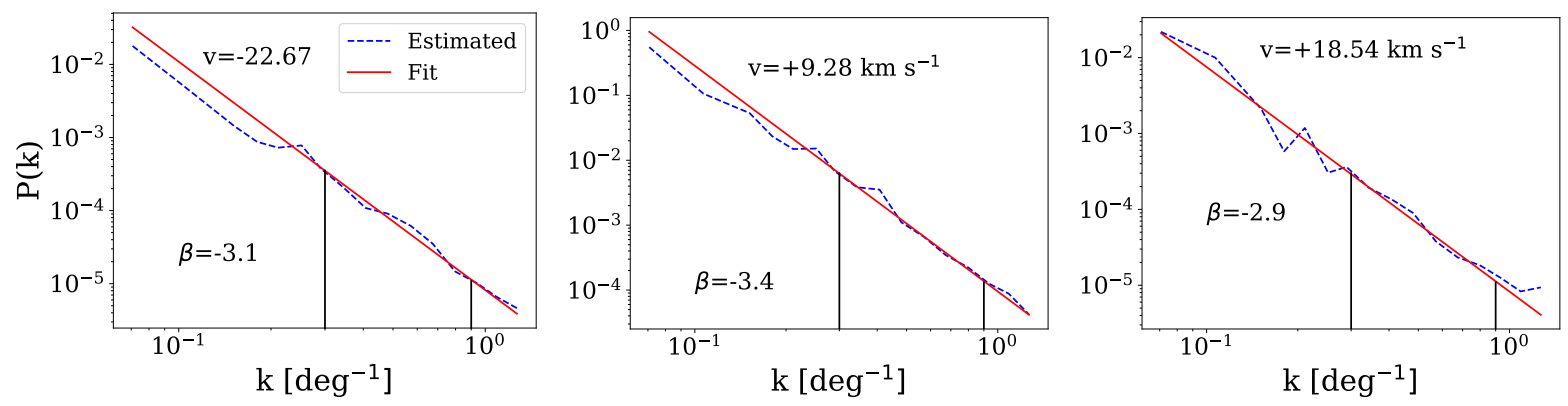

Figure 3. The estimated $P(k)$ for three representative velocity channels $-22.67,+9.28$ and $+18.54 \mathrm{~km} \mathrm{~s}^{-1}$ which are dominated by IVC, CNM and WNM respectively (blue dotted lines). The red lines show the best-fit power law model $P^{M}(k)$. The $k$ range we used for fitting is 0.3 to $0.9 \mathrm{deg}^{-1}$ which are shown by two vertical lines. The values of the power law index $(\beta)$ are shown in the lower left corner of each panel.

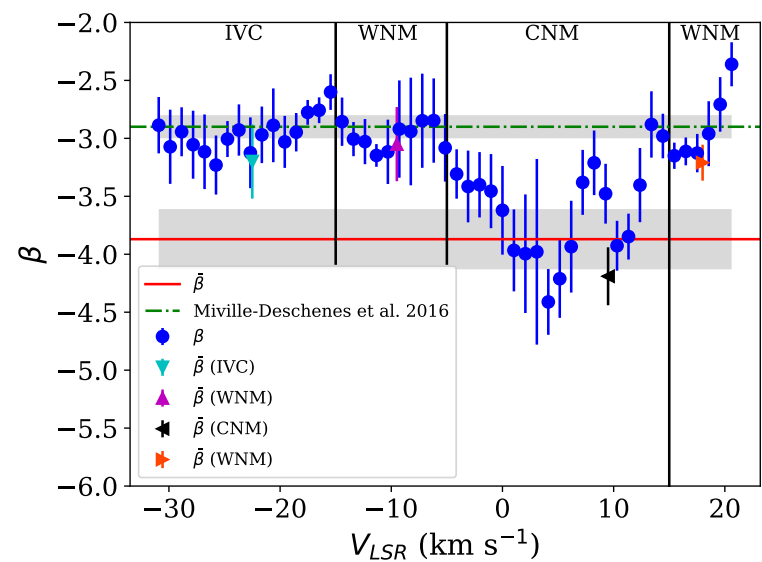

Figure 4. The variation of power law index, $\beta$ with $1 \sigma$ error bar as a function of LSR velocity $\left(v_{L S R}\right)$ (blue points). The power-law index, $\bar{\beta}$ estimated from integrated column density map for the IVC, WNM (negative velocity range), CNM and WNM (positive velocity range) are shown by cyan down, magenta up, black left and orange right triangle respectively. The green dash-dot line with shaded region is the measurement from Miville-Deschênes et al. (2016). The red solid line with shaded region shows the measured $\beta$ estimated from the integrated $\mathrm{H}$ I emission over the full velocity range of -30 to $+20 \mathrm{~km} \mathrm{~s}^{-1}$.

tween -2.6 to -3.2 for IVC which dominates in the velocity range from -30 to $-15 \mathrm{~km} \mathrm{~s}^{-1}$. The integrated column density map for this velocity range shown in Figure 5 (a). The integrated column density map is same as the average H I $21 \mathrm{~cm}$ brightness temperature map just apart from a constant factor. The value of $\bar{\beta}$ estimated using this integrated map for IVC is $-3.2 \pm 0.32$ (cyan down triangle in Figure 4). Martin et al. (2015) found the power-law index for IVC is around $-2.69 \pm 0.04$ which is slightly flatter as compared to our results. In the WNM dominated region $\left(-14<v_{L S R}<-5 \mathrm{~km} \mathrm{~s}^{-1}\right)$ the values of $\beta$ varies in the range -2.8 to -3.1 which is quite similar as shown for IVC. The integrated column density map for the WNM dominated region is shown in Figure 5 (b). The slope $\bar{\beta}$ estimated for this image is $-3.05 \pm 0.32$ which is (magenta up triangle in Figure 4 also similar as IVC.

The statistical properties of the CNM which dominate in the velocity range from -4 to $+15 \mathrm{~km} \mathrm{~s}^{-1}$ is quite different as shown for the IVC and WNM. In this case, the $\beta$ values vary in the range -2.9 to -4.4 . The integrated map for CNM is shown in Figure 5 (c) and corresponding $\bar{\beta}$ is shown by black left triangle which has a value $-4.19 \pm 0.25$. Here, the slope is much steeper as compared to the IVC and CNM. The velocity range +16 to $+20 \mathrm{~km} \mathrm{~s}^{-1}$ again dominated by the WNM and the integrated map is shown in Figure 5 (d). In this case, the value of $\bar{\beta}$ is $-3.21 \pm 0.15$ which is shown by the orange right triangle in Figure 4). We see that the measured $\beta$ in the CNM dominated velocity region are quite different from that of the WNM and IVC. This may be indicative of different nature of turbulence in different thermal phases depending on temperature, density and ionization fraction.

The power spectrum for the CNM is already measured earlier, and its power-law index varies in the range -2 to -3 (Kalberla \& Kerp 2016, Kalberla et al. 2017, Blagrave et al. 2017). Dickey et al. (2001) have measured the power spectrum of the warm HI gas and found power law index varies in the range -3 to -4 . Miville-Deschênes et al. (2003) have estimated the power spectrum of the Ursa Major highlatitude cirrus and found power law index with a value of $-3.6 \pm 0.2$. These studies suggest that the turbulent power spectra cannot be described by a unique power law.

We have also studied the statistical properties of the integrated emission for the whole velocity range from -30 to $+20 \mathrm{~km} \mathrm{~s}^{-1}$ and the corresponding image is shown in Figure 5 (e). Using the power spectrum estimated for this image, we get the best fit value of $\bar{\beta}$ to be $-3.87 \pm 0.26$ which is shown by a red solid line with shaded region. Based on dust continuum emission, Miville-Deschênes et al. (2016) estimated the value of $\bar{\beta}$ to be $-2.9 \pm 0.1$ which is shown with a green dash-dot line and a shaded region in Figure 4 The power law index derived using the $\mathrm{H}$ I $21 \mathrm{~cm}$ emission from the full range of the velocity is quite different with the value derived by Miville-Deschênes et al. (2016) for dust emission.

In Figure 6 we show the measured $P(\mathrm{k})$ with solid magenta lines for 20 velocity channels selected in the range from -30 to $+20 \mathrm{~km} \mathrm{~s}^{-1}$. Here, we also show two model $P^{M}(\mathrm{k})$ with power-law index -3.0 (red dash-dot line) and -4.2 (green dash line) which are the value of $\bar{\beta}$ similar to the WNM (also IVC) and CNM dominated region respectively. In the first row, we show five different velocity 


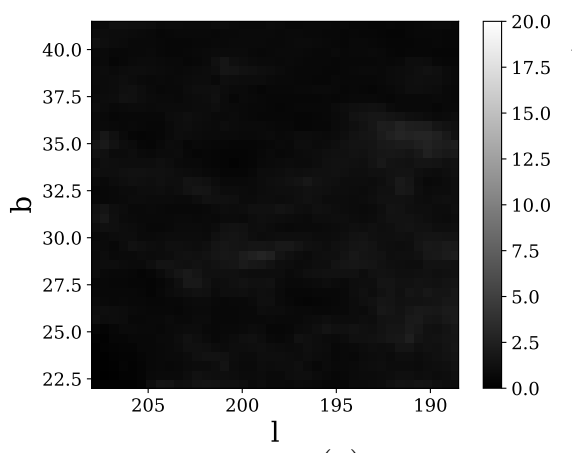

(a)

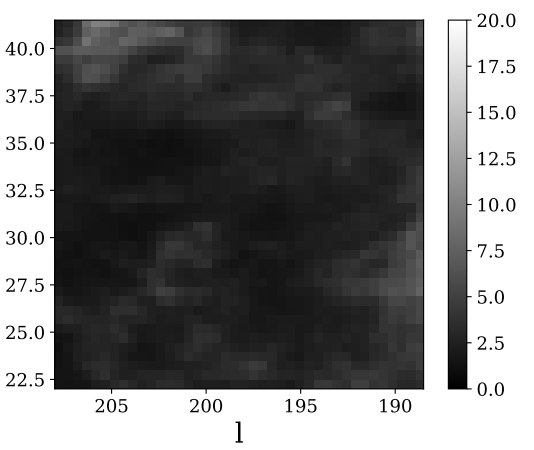

(b)

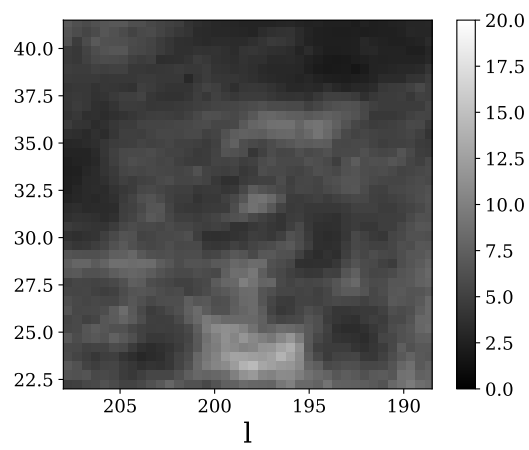

(c)

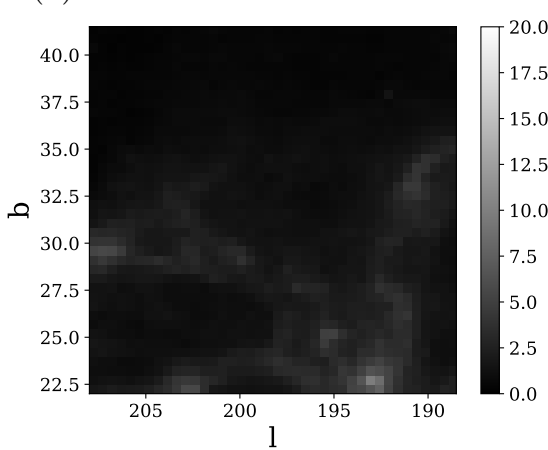

(d)

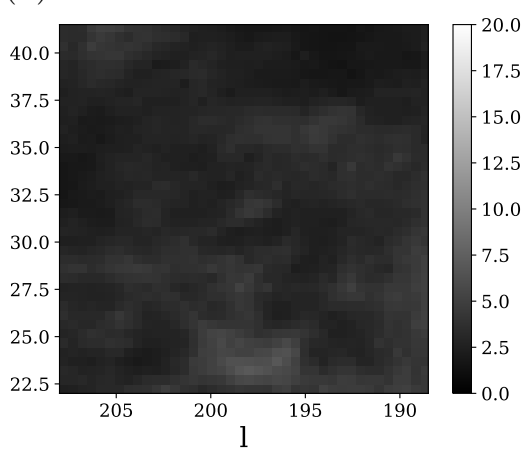

(e)

Figure 5. The average H I $21 \mathrm{~cm}$ brightness temperature maps which is proportinal to the atomic hydrogen column density $\left(N_{H I}\right)$ in different velocity range: (a) the IVC dominated region $\left(-30 \leq v_{L S R} \leq-15 \mathrm{~km} \mathrm{~s}^{-1}\right)$ (b) the WNM (negative velocity range) $(-14 \leq$ $\left.v_{L S R} \leq-5 \mathrm{~km} \mathrm{~s}^{-1}\right)$ (c) the CNM $\left(-4 \leq v_{L S R} \leq+15 \mathrm{~km} \mathrm{~s}^{-1}\right)(\mathrm{d})$ the WNM (positive velocity range) $\left(+16 \leq v_{L S R} \leq+20 \mathrm{~km} \mathrm{~s}{ }^{-1}\right)$ (e) the whole velocity range $\left(-30 \leq v_{L S R} \leq+20 \mathrm{~km} \mathrm{~s}^{-1}\right)$.

channels from IVC dominated regions. Similarly, the second, third and fourth rows are for the WNM (negative velocity region), CNM and WNM (positive velocity region) respectively. We see that the measured $P(\mathrm{k})$ in the IVC and WNM (both velocity ranges) dominated regions matches the $P^{M}(\mathrm{k})$ with $\bar{\beta}=-3.0$, whereas in the CNM dominated regions it matches with $\bar{\beta}=-4.2$.

Next, we have estimated $P(\mathrm{k})$ after averaging the velocity channels to a width ranging from 1.03 to $13.09 \mathrm{~km} \mathrm{~s}^{-1}$. Lazarian \& Pogosyan (2000) have shown that the intensity power spectrum may be changed due to velocity structure in the field. For "thick" slices, which are wider than the turbulent velocity dispersion, all the velocity information get averaged out and the measured $P(\mathrm{k})$ only quantify the density fluctuations in that field. However, for "thin" velocity slices, the measured $P(\mathrm{k})$ becomes shallower due to the velocity structure present in the field. The power law index of the $P(\mathrm{k})$ for thin slices becomes $n+\gamma / 2$, where $n$ is the power law index for thick slices and $\gamma$ is the power law index for the velocity structure function. The turbulent velocity dispersion for the cold Galactic HI gas is around $4.0 \mathrm{~km} \mathrm{~s}^{-1}$ (Radhakrishnan et al. 1972). The velocity width for the CNM we get is almost $5.4 \mathrm{~km} \mathrm{~s}^{-1}$ (Figure 1) which is slightly higher than the typical velocity dispersion of cold gas. In this study, we considered the velocity width from 1.03 to $13.09 \mathrm{~km} \mathrm{~s}^{-1}$. We can not probe the structures of velocity width below $1.25 \mathrm{~km} \mathrm{~s}^{-1}$ as we are limited by the resolution of the LAB survey. We expect the change in the power law index for thin slices of a width smaller than velocity dispersion. In Figure 7 we show the measured $P(k)$ only for three velocity width 1.03 (red solid line) 5.15 (blue dashed line) and $13.39 \mathrm{~km} \mathrm{~s}^{-1}$ (green dash-dot line) for clear visualization. Also, we show only four representative velocity channels $\left(v_{L S R}=-22.67,-9.28,+9.28\right.$ and $\left.18.54 \mathrm{~km} \mathrm{~s}^{-1}\right)$ in this figure, even if the exercise is repeated for all channels (and the results are the same for all the channels). We choose these four velocity channels from four different regions. Clearly, we do not observe any significant difference in the power spectrum after velocity channel averaging. This signifies that the fluctuations are mainly dominated by the density fluctuations. We use the maximum error in measured $\beta(\sim 0.8)$ to constrain the value of $\gamma$ to be $0.0 \pm 1.1$ which is consistent with the predicted Kolmogorov turbulence $(\gamma=2 / 3)$ and also with a shock-dominated medium $(\gamma=1.0)$.

\section{SUMMARY AND CONCLUSIONS}

Measurements of $\mathrm{H}$ I $21 \mathrm{~cm}$ power spectrum, $P(\mathrm{k})$ can be used to quantify the small-scale structures within our Galaxy. In this paper, we have studied the $21 \mathrm{~cm} P(\mathrm{k})$ over a length scale ranging from $3 \mathrm{pc}$ to $50 \mathrm{pc}$. We have used high-resolution LAB survey data to measure the $P(\mathrm{k})$ of a $20^{\circ} \times 20^{\circ}$ region centred at the Galactic coordinate $\left(198^{\circ}, 32^{\circ}\right)$. Here, we have considered a velocity range of -30 to $+20 \mathrm{~km} \mathrm{~s}^{-1}$ which we believe to be dominated by $21 \mathrm{~cm}$ signal only. The non-detection of molecular ${ }^{12} C O(J=1-0)$ emission in this field (Miville-Deschênes et al. 2016) also indicates the same. Miville-Deschênes et al. (2016) have al- 

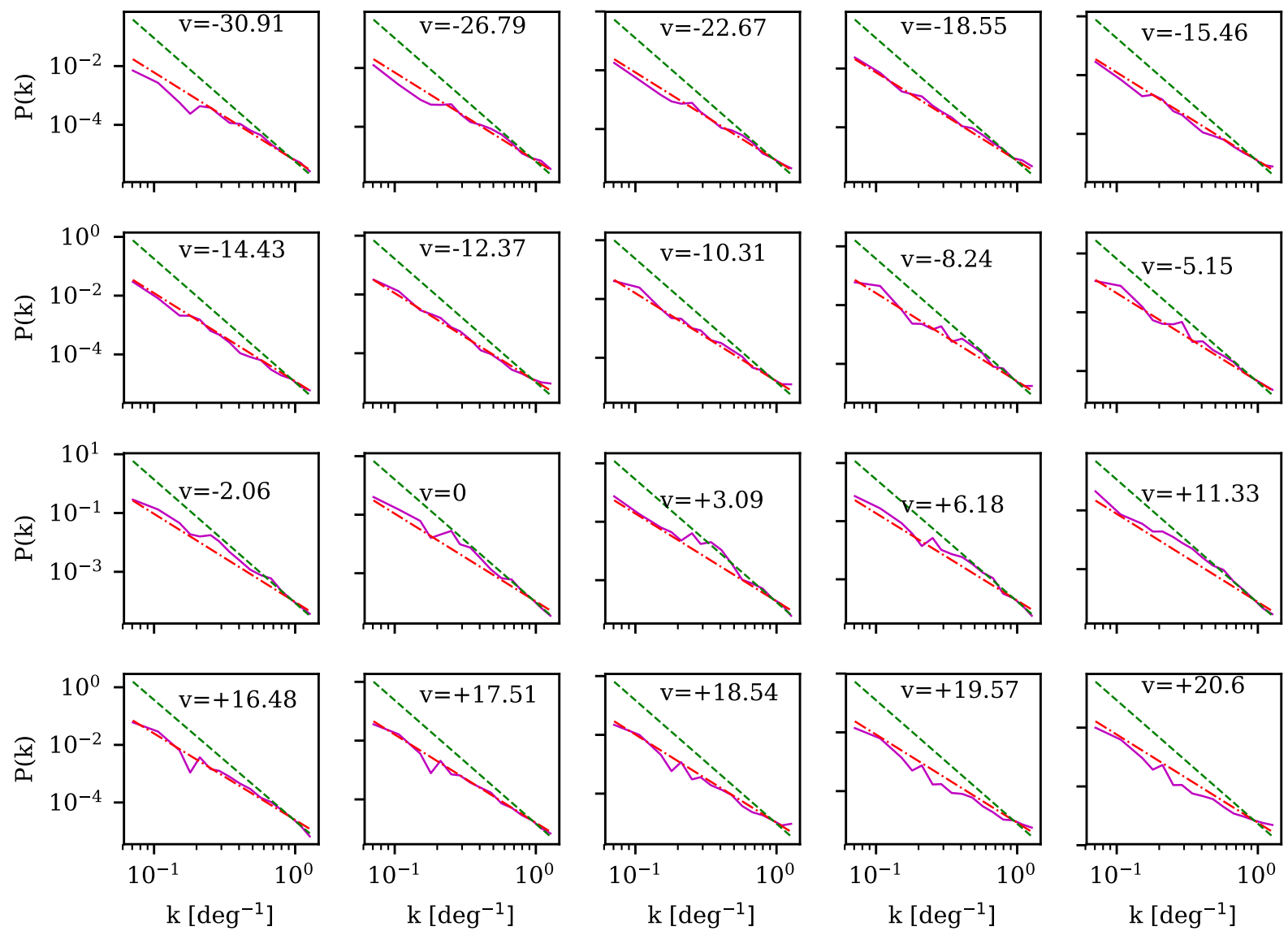

Figure 6. The measured $21 \mathrm{~cm} P(\mathrm{k})$ (megenta solid line) for 20 different velocity channels in the range from -30 to $+20 \mathrm{~km} \mathrm{~s}{ }^{-1}$. The green dash lines shows the model $P^{M}(\mathrm{k})$ with slope -3.0 and the red dash-dot line is for -4.2 which are the value of $\bar{\beta}$ similar to the WNM (also IVC) and CNM dominated region respectively. Each row shows five different velocity channels for differnt components: First row (IVC), Second row (WNM in negative velocity range), Third row (CNM), Fourth row (WNM in positive velocity range). The power spectrum for the WNM and IVC dominated region matches model $P^{M}(\mathrm{k})$ with $\beta=-3.0$, whereas the CNM dominated region matches with $\beta=-4.2$.

ready measured the $P(\mathrm{k})$ of continuum dust emission in this region using the emission maps from three different surveys. They found $P(\mathrm{k})$ follows a power law with index $-2.9 \pm 0.1$. We see that the measured $21 \mathrm{~cm} P(\mathrm{k})$ also follows a power law within the scale range probed here. However, the power law index was found to have a significant channel to channel variation over this velocity range. Also, the best fit value of the power-law index $\bar{\beta}$ estimated from the integrated map from different regions in this velocity ranges are quite different. The values of $\bar{\beta}$ are $-3.2 \pm 0.32,-3.05 \pm 0.32,-4.19 \pm 0.25$ and $-3.21 \pm 0.15$ for the IVC, WNM (negative velocity range), CNM and WNM (positive velocity range) dominated regions respectively. We see that the measured $\bar{\beta}$ in the $\mathrm{CNM}$ dominated region is significantly different as compared to other components e.g. IVC, WNM. A possible interpretation of this can be an inherent quantitative difference of the turbulence power spectra for different phases of the ISM. There is no a priori reason to assume that the nature of turbulence identical in these phases. In the absence of such universality, the measured $P(\mathrm{k})$ may vary depending on the relative contribution of these phases.

Please note that, while modelling the observed power spectrum, we assume a power law form based on the fact that small-scale structures generated due to turbulence will have such scaling between the injection scale and the dissipation scale. For Kolmogorov-like turbulence in the neutral gas, the Reynold's number is given by $R_{e}=3 \times$ $10^{4} \frac{L}{10 \mathrm{pc}} \frac{v}{10 \mathrm{~km} \mathrm{~s}^{-1}} \frac{n}{1 \mathrm{~cm}^{-3}}$ and the dissipation scale is $l_{d}=$ $L R_{e}^{-3 / 4}$ where $v$ is the turbulent velocity dispersion, $n$ is the average density at the energy dissipation scale, and $L$ is the energy injection scale for turbulence (Subramanian 1998). If we consider the two components with $\sigma_{v}=5.4$ and $17.3 \mathrm{~km} \mathrm{~s}^{-1}$ to be cold and warm neutral medium respectively, with typical parameters like $T_{k}=100$ and $5000 \mathrm{~K}$, and density in the range of $10-100$ and $0.1-1 \mathrm{~cm}^{-3}$ for these two phases, the dissipation scales turn out to be $2.23 \times 10^{-4}$ $-1.25 \times 10^{-3}$ pc for CNM and $3.07 \times 10^{-3}-1.73 \times 10^{-2}$ pc for WNM, well below the $3.4 \mathrm{pc}$ scale probed here using LAB data.

We have also studied the power spectrum after averaging the velocity of width ranging from 1.03 to $13.39 \mathrm{~km} \mathrm{~s}^{-1}$. For thin velocity slices, which are smaller than the turbulent velocity dispersion $\left(4.0 \mathrm{~km} \mathrm{~s}^{-1}\right)$, the measured $P(k)$ 

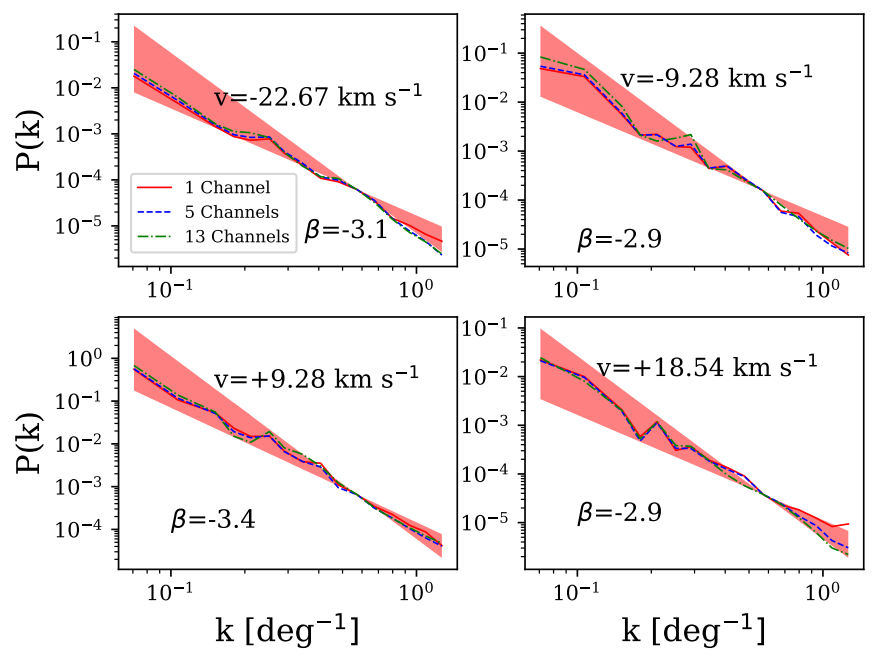

Figure 7. The estimated $P(\mathrm{k})$ after averaging different velocity channels. The red solid, blue dashed and green dash-dot are for $1.03,5.15$ and $13.39 \mathrm{~km} \mathrm{~s}^{-1}$ respectively for four representative velocity channels taken from four different regions. The shaded regions show the area for an uncertainty of \pm 0.8 in the power law index $\beta$.

becomes shallower due to the velocity fluctuations present in the field (Lazarian \& Pogosyan 2000). However, we do not observe any significant difference in measured $P(\mathrm{k})$ after velocity channel averaging. This implies that either the transition happens below $1.25 \mathrm{~km} \mathrm{~s}^{-1}$ which is the resolution of the LAB survey, or the velocity structure function is shallower than our measurement error of the power law index of the power spectra. From this study, we can only constrain the value of $\gamma$ to be $0.0 \pm 1.1$ which is consistent with the predicted Kolmogorov turbulence $(\gamma=2 / 3)$ and also with a shock-dominated medium $(\gamma=1.0)$. We plan to investigate this issue with better spectral resolution data in future.

Finally, similar high-velocity resolution investigation of Galactic H I power spectra for different directions will be helpful to understand if the nature of turbulence is truly different for the cold and warm phases.

\section{ACKNOWLEDGEMENTS}

We thank an anonymous referee for helpful comments. SC acknowledges NCRA-TIFR for providing financial support. SC would like to thank Narendranath Patra for useful discussion. SC acknowledges NR's Infosys Young Investigator grant for supporting his collaborative visit to IISc and IISc for an opportunity to work on this project during the visit. NR acknowledges support from the Infosys Foundation through the Infosys Young Investigator grant.

\section{References}

Arnal, E. M., Bajaja, E., Larrarte, J. J., Morras, R., \& Pöppel, W. G. L. 2000, Astronomy and Astrophysics Supplement Series, 142, 35
Bajaja, E., Arnal, E. M., Larrarte, J. J., et al. 2005, A \& A, 440, 767

Blagrave, K., Martin, P. G., Joncas, G., et al. 2017, ApJ, 834,126

Crovisier, J., \& Dickey, J. M. 1983, A \& A, 122, 282

Crovisier, J., Dickey, J. M., \& Kazes, I. 1985, A \& A, 146, 223

Davis, R. J., Diamond, P. J., \& Goss, W. M. 1996, MNRAS, 283,1105

Deshpande, A. A., McCulloch, P. M., Radhakrishnan, V., \& Anantharamaiah, K. R. 1992, MNRAS, 258, 19P

Deshpande, A. A., Dwarakanath, K. S., \& Goss, W. M. 2000, ApJ, 543, 227

Deshpande, A. A. 2000, MNRAS, 317, 199

Dickey, J. M., McClure-Griffiths, N. M., Stanimirović, S.,

Gaensler, B. M., \& Green, A. J. 2001, ApJ, 561, 264

Dieter, N. H., Welch, W. J., \& Romney, J. D. 1976, ApJL, 206, L113

Dutta, P., Chengalur, J. N., Roy, N., et al. 2014, MNRAS, 442,647

Field, G. B., Goldsmith, D. W., \& Habing, H. J. 1969, ApJL, 155, L149

Field, G. B. 1973, Molecules in the Galactic Environment, 21

Frail, D. A., Weisberg, J. M., Cordes, J. M., \& Mathers, C. 1994, ApJ, 436, 144

Frigo, M., \& Johnson, S. G. 2005, Proceedings of the IEEE, 93, 216

Green, D. A. 1993, MNRAS, 262, 327

Hartmann, D., \& Burton, W. B. 1997, Atlas of Galactic Neutral Hydrogen, by Dap Hartmann and W. Butler Burton, pp. 243. ISBN 0521471117. Cambridge, UK: Cambridge University Press, February 1997., 243

Hennebelle, P., \& Audit, E. 2007, A \& A, 465, 431

HI4PI Collaboration, Ben Bekhti, N., Flöer, L., et al. 2016, A \& A, 594, A116

Johnston, S., Koribalski, B., Wilson, W., \& Walker, M. 2003, MNRAS, 341, 941

Kalberla, P. M. W., Schwarz, U. J., \& Goss, W. M. 1985, A \& A, 144, 27

Kalberla, P. M. W., Burton, W. B., Hartmann, D., et al. 2005, A \& A, 440, 775

Kalberla, P. M. W., \& Kerp, J. 2016, A \& A, 595, A37

Kalberla, P. M. W., Kerp, J., Haud, U., \& Haverkorn, M. 2017, A \& A, 607, A15

Lazarian, A., \& Pogosyan, D. 2000, ApJ, 537, 720

Martin, P. G., Blagrave, K. P. M., Lockman, F. J., et al. 2015, ApJ, 809, 153

Miville-Deschênes, M.-A., Joncas, G., Falgarone, E., \& Boulanger, F. 2003, A \& A, 411, 109

Miville-Deschênes, M.-A., Duc, P.-A., Marleau, F., et al. 2016, A \& A, 593, A4

Nagashima, M., Inutsuka, S.-i., \& Koyama, H. 2006, ApJL, 652, L41

Radhakrishnan, V., Murray, J. D., Lockhart, P., \& Whittle, R. P. J. 1972, ApJS, 24, 15

Roy, N., Chengalur, J. N., Dutta, P., \& Bharadwaj, S. 2010, MNRAS, 404, L45

Roy, N., Minter, A. H., Goss, W. M., Brogan, C. L., \& Lazio, T. J. W. 2012, ApJ, 749, 144

Sofue, Y. 2013, PASJ, 65, 118

Stanimirović, S., Weisberg, J. M., Pei, Z., Tuttle, K., \& 
Green, J. T. 2010, ApJ, 720, 415

Subramanian, K. 1998, MNRAS, 294, 718

Vázquez-Semadeni, E., Ryu, D., Passot, T., González,

R. F., \& Gazol, A. 2006, ApJ, 643, 245 